\title{
Diffuse involvement of the leptomeninges by tumour-a clinical and pathological study of 63 cases
}

\author{
RICHARD BOYLE* \\ M.B. B.S., F.R.A.C.P. \\ MYFANWY ThOMAS* \\ M.B. B.S., M.R.C.P., D.C.H. \\ J. Hume Adams $\dagger$ \\ M.B. Ch.B., Ph.D., F.R.C.Path., F.R.C.P. \\ *Department of Neurology, and †University Department of Neuropathology, \\ Institute of Neurological Sciences, Southern General Hospital, Glasgow
}

\begin{abstract}
Summary
The clinical and pathological features of 63 cases of post-mortem-proved diffuse infiltration of the leptomeninges by tumour are presented. A wide variety of tumours of the nervous system, both primary and secondary, was found to give rise to such involvement, with adenocarcinoma having a particular propensity to behave in this manner. Dysfunction of cranial and spinal nerves, a confusional state and headache were prominent clinical features. Examination of the cerebro-spinal fluid was found to be less useful diagnostically in cases of primary tumours of the nervous system with leptomeningeal involvement than in cases of diffuse infiltration of the meninges by carcinoma.
\end{abstract}

\section{Introduction}

Diffuse infiltration of the leptomeninges by malignant primary tumours of the central nervous system (CNS) or by metastatic carcinoma is a well recognized entity. It is, however, uncommon and the clinical features are so protean that, with the possible exception of medulloblastoma which characteristically spreads throughout the subarachnoid space, it may be difficult to achieve the correct diagnosis during life. With the aim of promoting a greater awareness of this syndrome, the authors wish to review briefly the principal clinical and pathological features in 63 cases.

\section{Material and methods}

During the period 1964-1978, 63 cases of diffuse infiltration of the leptomeninges by tumour were encountered in the Department of Neuropathology of the Institute of Neurological Sciences, Glasgow. Excluded from this series are cases in which the primary tumour was medulloblastoma and cases of meningeal leukaemic infiltration. Cases in which there was only local invasion of the meninges from an adjacent tumour are also excluded.

Since it seemed likely that the presence of mass tumour deposits within the brain or spinal cord could affect the clinical presentation, the cases were divided into the following groups.

\section{Group 1}

Patients with primary tumours of the CNS or nerve roots ( 23 cases).

(a) Those with macroscopic deposits of tumour in the CNS or spinal nerve roots (18 cases, Table 1$)$. The average age of the males was 39 years and of the females 32 years.

(b) Those without macroscopic deposits of tumour in the CNS or spinal nerve roots (5 cases, Table 2). The ages ranged from 14 months to 63 years.

TABle 1. Group 1a

\begin{tabular}{lcc}
\hline \multirow{2}{*}{\multicolumn{1}{c}{ Tumour type }} & \multicolumn{2}{c}{ No. of cases } \\
\cline { 2 - 3 } & Male & Female \\
\hline Astrocytoma & 5 & 2 \\
Gliosarcoma & - & 1 \\
Oligodendroglioma & 1 & - \\
Ependymoma & 2 & - \\
Neurofibrosarcoma & - & 2 \\
Microglioma & 1 & 1 \\
Spinal malignant blue naevus & - & 1 \\
Primitive neuro-ectodermal tumour & - & 1 \\
Choroid plexus malignancy & - & 1 \\
Total & 9 & 9 \\
\hline \multicolumn{1}{c}{ TABLE 2. Group } & $1 \mathrm{~b}$ & \\
Diffuse meningeal astrocytoma & 1 & 1 \\
Primary melanoma of meninges & 1 & -1 \\
Neurocutaneous melanosis & 1 & 1 \\
Total & 3 & 2 \\
\hline
\end{tabular}

\section{Group 2}

Patients with primary tumours outside the CNS (40 cases).

(a) Those with macroscopic metastases in the CNS (22 cases, Table 4). The average age of the males was 59 years and of the females 58.5 years. 
(b) Those without macroscopic metastases in the CNS (18 cases, Table 5). The average age of the males was 56 years and of the females 57 years.

The clinical records of the patients were analysed to determine retrospectively if there were clinical features suggestive of the presence of diffuse meningeal tumour which might have led to an earlier correct diagnosis. Particular attention was paid to Groups $1 \mathrm{~b}$ and $2 \mathrm{~b}$ where the clinical picture was not complicated by a focal mass lesion within the substance of the brain or spinal cord.

A full post-mortem was performed in every case and the brains were suspended in $10 \%$ formalin for at least 3 weeks before dissection. Not every spinal cord was available since some of the brains were referred from other hospitals for neuropathological assessment. After transecting the midbrain, the cerebral hemispheres were cut in the coronal plane into slices $10 \mathrm{~mm}$ thick. The cerebellum was cut at right angles to the folia, and the brain stem in the horizontal plane. Numerous representative blocks were taken for histological examination from any macroscopically identifiable tumour and from the hypothalamus, cerebral cortex, cerebellum, brain stem and (where available) the spinal cord to assess the extent and distribution of diffuse meningeal tumour.

\section{Clinical features \\ Group 1}

Those presenting clinical features clearly due to malignant infiltration of the meninges and not related to the presence of a mass lesion are sum- $\frac{2}{3}$ marized in Table 3. This table includes the clinical $\stackrel{\Phi}{\propto}$ features of 7 cases in Group 1a (3 cases of astro- $c$. cytoma and one each of gliosarcoma, microglioma, $\ddot{\vec{F}}$ choroid plexus papilloma and primitive neuro- $\stackrel{S}{+}$ ectodermal tumour), and of the 5 cases in Group $1 \mathrm{~b}$. In one case in Group $1 \mathrm{~b}$ the clinical picture was $\frac{\bar{\sigma}}{\bar{N}}$ dominated by the presence of a large melanoma $\frac{\bar{s}}{\overrightarrow{0}}$ arising from the leptomeninges and compressing the $\propto$ underlying temporal lobe.

It can be seen that the clinical features can be $\vec{A}$ broadly divided into 4 groups: (1) manifestations suggesting raised intracranial pressure without focal $\vec{\omega}$ features (e.g. increase in head size, headache, $\stackrel{\circ}{\circ}$ vomiting, drowsiness and papilloedema). These $\frac{0}{7}$ features were shown subsequently either radio- 3 . logically or at post-mortem to be associated in this group with the presence of communicating hydrocephalus; (2) manifestations of meningeal irritation; $\omega$ (3) manifestations of cranial nerve dysfunction, and $\vec{f}$ (4) manifestations of spinal cord or spinal nerve 6 involvement.

The clinical progression in these cases, from the $\vec{z}$ time of the first presentation with features of meningeal involvement, suggested local spread of the $\frac{\bar{S}}{\partial}$ meningeal infiltrate rather than widespread exten- $\vec{\Im}$ sion. In one case there was clinical evidence of both intracranial and spinal meningeal involvement at the time of initial presentation.

Cerebrospinal fluid $(C S F)$. The results of examiñ ation of the CSF were available in 14 of the $23 \frac{\bar{\partial}}{0}$ cases. Malignant cells were found in only 3 cases:

TABLE 4. Group 2a

\begin{tabular}{|c|c|c|c|c|}
\hline \multirow[b]{2}{*}{ Site of primary tumour } & \multicolumn{2}{|c|}{ No. of cases } & \multirow{2}{*}{$\begin{array}{l}\text { Primary tumour known at time } \\
\text { of clinical presentation }\end{array}$} & \multirow{2}{*}{$\begin{array}{l}\text { Mean survival } \\
\text { (months) }\end{array}$} \\
\hline & Male & Female & & \\
\hline Lung & 8 & 2 & 1 & $1 \cdot 7$ \\
\hline Breast & - & 3 & 3 & 4.0 \\
\hline Melanoma & 1 & 2 & 2 & $1 \cdot 3$ \\
\hline Stomach & 1 & 1 & 1 & $2 \cdot 0$ \\
\hline Prostate & 1 & - & - & $5 \cdot 0$ \\
\hline Kidney & 1 & - & - & $2 \cdot 0$ \\
\hline Non-Hodgkin's lymphoma & - & 1 & 1 & $2 \cdot 5$ \\
\hline No primary tumour found & - & 1 & - & $2 \cdot 5$ \\
\hline Total & 12 & 10 & 8 & $2 \cdot 3$ \\
\hline
\end{tabular}

TABLE 5. Group 2b

\begin{tabular}{lccccc}
\hline & \multicolumn{2}{c}{ No. of cases } & & Primary tumour known at time \\
Site of primary tumour & Male & Female & $\begin{array}{c}\text { Mean survival } \\
\text { (months) }\end{array}$ \\
\cline { 2 - 5 } Lung & 6 & - & 1 & $4 \cdot 0$ \\
Stomach & 5 & 1 & 4 & $2 \cdot 2$ \\
Breast & - & 4 & -1 & $2 \cdot 5$ \\
Parathyroid & 1 & - & 7 & $0 \cdot 2$ \\
Non-Hodgkin's lymphoma & - & 1 & $6 \cdot 0$ \\
Total & 12 & 6 & & $2 \cdot 8$ \\
\hline
\end{tabular}


TABle 3. Group 1. Presenting features-11 cases

\begin{tabular}{lclc}
\hline Symptoms & No. of cases & \multicolumn{1}{c}{ Signs } & No. of cases \\
\hline Headache & 6 & Nuchal rigidity & 7 \\
Weakness of legs & 4 & Areflexia \pm weakness of legs & 5 \\
Vomiting & 3 & Papilloedema & 5 \\
Diplopia & 2 & Sensory loss in legs & 3 \\
Low back pain & 1 & Cranial nerve palsies & 2 \\
Pain in legs & 1 & (III, 1; VI, 1; VII, 2; X, 1; & \\
Dysphagia & 1 & XII, 1) & 2 \\
Blurred vision & 1 & Spastic paraparesis & 2 \\
Seizures & 1 & Incontinence of urine & 2 \\
& & Drowsiness & 2 \\
& & Confusion & 1 \\
& & Increasing head size & 1 \\
\hline
\end{tabular}

one of oligodendroglioma, one of ependymoma and one of neurocutaneous melanosis. The total cell count was elevated in 5 cases (maximum cell count $100 / \mathrm{mm}^{3}$ ), including the 3 in which malignant cells were present, and the cells were predominantly mononuclear. CSF protein was elevated above $0.5 \mathrm{~g} / 1$ in 12 cases, with a level of $64 \mathrm{~g} / 1$ being the highest recorded (a case of ependymoma). CSF glucose was depressed below $2 \mathrm{mmol} / \mathrm{l}$ in only 2 cases.

Prognosis. The mean survival time from the onset of clinical features related to meningeal infiltration was 6.8 weeks. In Group 1a the mean survival time from the onset of the first neurological symptom was 8.5 months, not different from survival times usually associated with malignant primary tumours of the CNS.

\section{Group 2}

Those presenting clinical features clearly due to malignant infiltration of the meninges and not related to the presence of metastatic mass lesions within the substance of the brain or cord are listed in Table 6 . This table includes the presenting clinical features of all 17 cases in Group 2b and of 6 cases in
Group $2 \mathrm{a}$ in which relevant clinical features were present ( 3 cases of carcinoma of the lung and one case each of carcinoma of the parathyroid, carcinoma of the stomach and non-Hodgkin's lymphoma). It can be seen that the majority of the presenting clinical features related to malignant infiltration of the meninges could be divided into the same 4 categories as in Group 1. A further conspicuous presenting feature in this group was a confusional state.

In 4 cases there was clinical evidence of both intracranial and spinal meningeal tumour at the time of initial presentation. In the majority of cases in this group the clinical course suggested local rather than widespread extension of the meningeal tumour.

In only 15 of these 40 cases was a primary neoplasm known to be present at the time of neurological presentation (Tables 4 and 5). Of these 15 cases, 7 were cases of carcinoma of the breast. In 8 of the 40 cases in Group 2, no secondary deposit outside the nervous system was discovered at postmortem, excluding regional lymph node involvement.

Cerebrospinal fluid. The results of examination of the CSF were available in 16 of these 40 cases. Of these 16 , malignant cells were identified in 6 . The total cell count was elevated in 15 cases (maximum

TABLE 6. Group 2. Presenting features -23 cases

\begin{tabular}{lclc}
\hline \multicolumn{1}{c}{ Symptoms } & No. of cases & \multicolumn{1}{c}{ Signs } & No. of cases \\
\hline Headache & 7 & Cranial nerve palsies & 12 \\
Weakness of legs & 4 & [III, 2; V, 3; VI, 2; VII, 6 (4 & \\
Low back pain & 4 & bilat); VIII, 7 (3 bilat); X, 6 (3 & \\
Pain in legs & 4 & bilat)] & 9 \\
Deafness & 3 & Confusion & 7 \\
Dysphagia & 3 & Areflexia 士 weakness of legs & 5 \\
Vomiting & 3 & Papilloedema & 5 \\
Dizziness & 3 & Nuchal rigidity & 3 \\
Diplopia & 3 & Sensory loss in legs & 3 \\
Blurred vision & 2 & Ataxia of gait & 1 \\
Unsteadiness of gait & 2 & Flaccid arm monoparesis & 1 \\
Hoarseness & 1 & Sensory loss in arms & 1 \\
Pain in arms & 1 & Nystagmus & \\
\hline
\end{tabular}


$770 / \mathrm{mm}^{3}$ ), the predominant cell type again being mononuclear. The protein levels were elevated above $0.5 \mathrm{~g} / \mathrm{l}$ in all cases (maximum $11.0 \mathrm{~g} / \mathrm{l}$ ) and the glucose was depressed below $2 \mathrm{mmol} / \mathrm{l}$ in 10 cases.

Prognosis. The mean survival times from the onset of neurological symptoms are shown in Tables 4 and 5 .

\section{Pathology}

\section{Group 1a (Table 1)}

This group of 18 cases of primary tumours of the nervous system could be further divided pathologically into 3 sub-groups.

There were 13 cases of conventional primary cerebral tumours. Seven of the tumours were astrocytomas, 6 of them being anaplastic. Four of these 7 astrocytomas originated near the subarachnoid space or the ventricular system-one in the medulla oblongata, one in the hypothalamus (Fig. 1a), one in the interventricular septum/corpus callosum and one in the left frontal lobe immediately adjacent to a

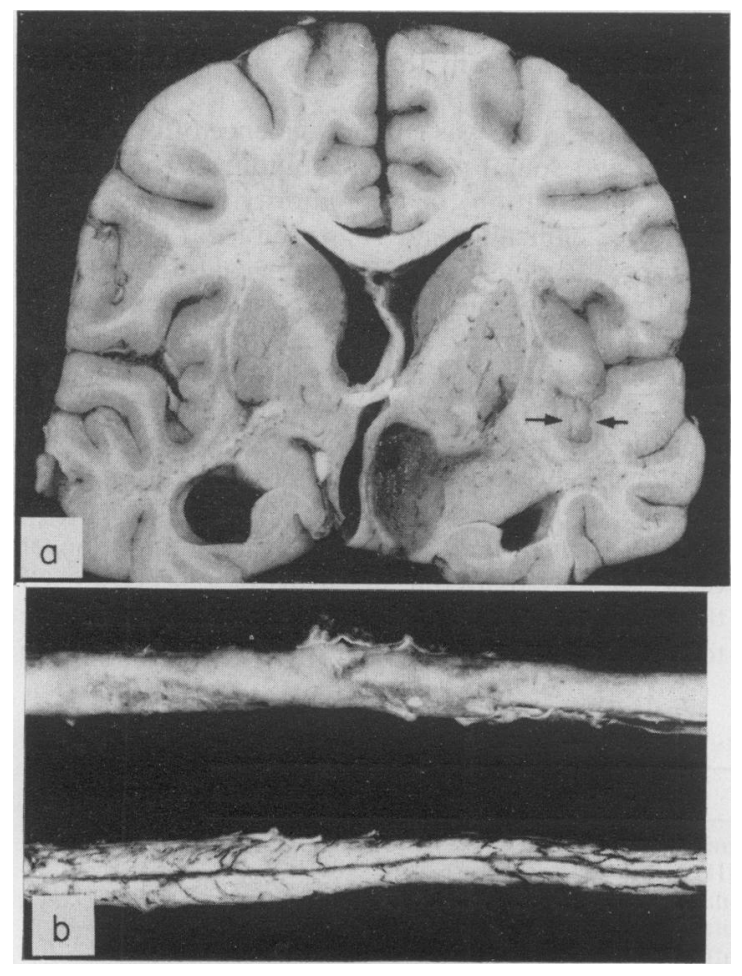

Fig. 1. Meningeal gliomatosis. Male aged 38: group 1a. (a) There is a well circumscribed astrocytoma in the posterior hypothalamic region adjacent to the third ventricle. Note the unusually thick sheet of tumour in the right Sylvian fissure (arrow). (b) Spinal cord from the same case and a normal cord (below) for comparison. The surface of the cord is obscured by a sheet of tumour.
Sylvian fissure. Three tumours in Group 1a arose within the ventricular system-2 ependymomas of the 4th ventricle and a papillary tumour of the choroid plexus arising in the right lateral ventricle. Of the remaining 3 , one was a relatively well differentiated oligodendroglioma, one a gliosarcoma $\bar{O}$ with tumour on either side of the insula (anaplastic astrocytoma in the temporal lobe and a fibrosarcoma in the frontal lobe), and there was one primitive neuro-ectodermal tumour in a 12-year-old female patient which originated in the pineal region and could not be classified precisely.

There were 2 examples of intrinsic lymphomas (microgliomatosis) of the CNS with no clinical or post-mortem evidence of systemic lymphoma. In one of these cases there was a discrete intradural spinal mass, while in the other case there were poorly defined masses of lymphoma in the hypothalamus and in the right temporal region.

There were 3 cases in which the tumour had arisen in the spinal nerve roots. Two of these presented originally as lumbar neurofibromas but when they recurred the appearance of the recurrent tumour was that of neurofibrosarcoma with diffuse meningeal infiltration. The third case was a rare example of a cervical spinal blue naevus with diffuse leptomeningeal infiltration.

\section{Group $1 b$ (Table 2)}

There were 5 cases in which the tumour had apparently arisen within the subarachnoid space. Two of these were examples of moderately well differentiated diffuse astrocytoma of the meninges with no identifiable tumour within the substance of the brain or spinal cord. There were 3 cases of diffuse primary malignant melanoma of the meninges; 2 of these were associated with multiple benign pigmented hairy naevi, constituting the syndrome of neurocutaneous melanosis, and in one of these there was a large tumour mass arising from the meninges and compressing the underlying temporal lobe.

\section{Group 2a (Table 4)}

Of the 22 cases in this group, the commonest site of the primary tumour was the bronchus (10 cases); 5 of these were adenocarcinomas, 3 were small cell anaplastic tumours and 2 were squamous carcinomas. In one of these cases (an apical adenocarcinoma) the tumour appeared to have gained access to the subarachnoid space by spreading proximally along the nerves of the brachial plexus.

Of the remaining 12 cases, the primary tumour was found in the breast in 3 ( 2 adenocarcinoma and one spheroidal cell carcinoma), in the stomach in 2 cases (both adenocarcinoma), in the prostate in one case (a conventional clear cell carcinoma) and in the 
kidney in one case (a nephroblastoma in a 10-monthold infant). There were 3 examples of metastatic melanoma, while in one case the primary tumour was not identified. There was one case of disseminated non-Hodgkin's lymphoma.

\section{Group $2 b$ (Table 5)}

The distribution of the primary tumour in the 18 cases in this group was essentially similar to that of Group 2a, apart from the higher incidence of primary tumours of the stomach-6 cases, 5 of which were adenocarcinoma and one an example of linitis plastica. There were 5 patients with primary bronchial tumours ( 3 adenocarcinomas and 2 anaplastic small cell tumours), 4 patients with primary tumours of the breast ( 2 adenocarcinomas and 2 spheroidal cell carcinomas), one patient with an alveolar cell carcinoma of the lung and one where the primary tumour appeared to have originated in a parathyroid gland. This last case was a difficult diagnostic problem because of the widespread dissemination of tumour but the consensus of opinion of a group of experienced general pathologists was that the primary tumour was a parathyroid carcinoma. There was one case of non-Hodgkin's lymphoma.

The distribution of the diffuse meningeal tumour

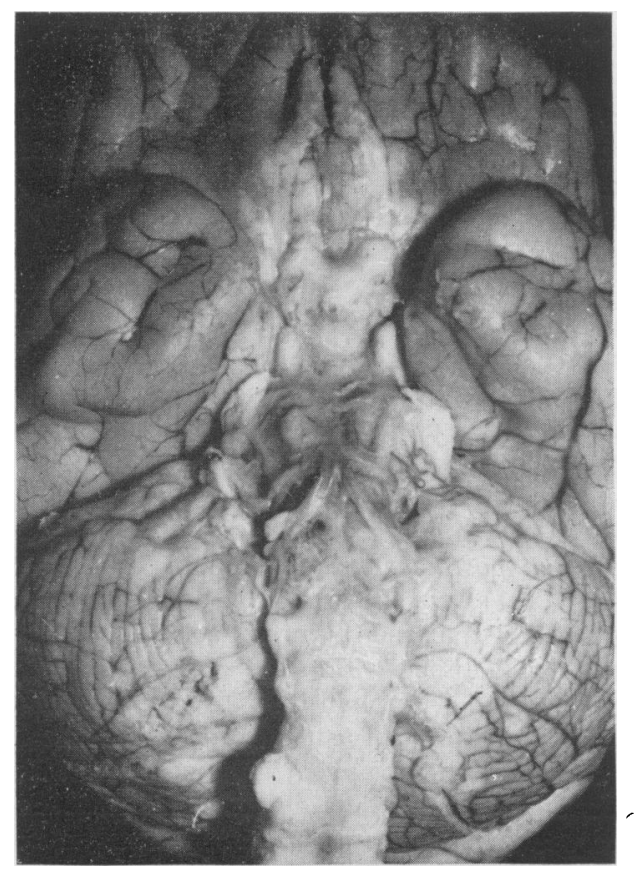

FIG. 2. Meningeal gliomatosis. Female aged 9: primary primitive neuroectodermal tumour arising in the pineal region: group 1a. Tumour is obscuring the optic chiasma, the hypothalamic region, the brain stem and the adjacent medial parts of the cerebellar hemispheres. in both groups 1 and 2 was essentially similar. There was a great tendency for the tumour to occur in the basal cisterns (Fig. 2), around the brain stem and spinal cord (Fig. 1b) and on the surface of the cerebellum. A particularly characteristic feature was the presence of small nodules of tumour on the nerve roots of the cauda equina (Fig. 3 ) with matting

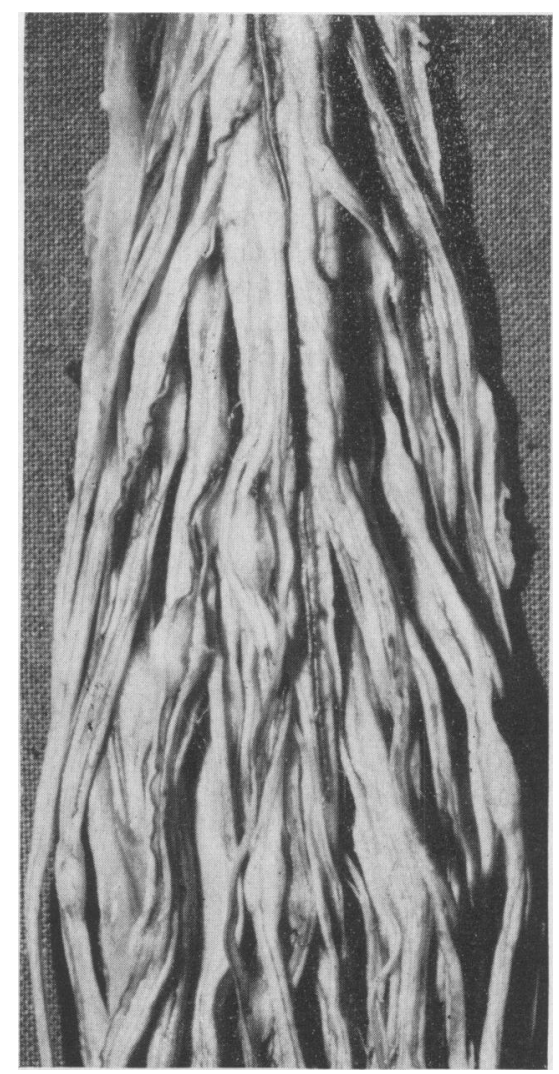

FIG. 3. Meningeal carcinomatosis. Male aged 70: carcinoma of bronchus: group $2 \mathrm{a}$. There are numerous nodules of tumour on the nerve roots of the cauda equina. The nerve roots were also loosely adherent to each other.

of the nerve roots. It should be emphasized that in 15 of the cases in Group 2 (8 in Group 2a and 7 in Group 2b) there was no macroscopic evidence of tumour in the subarachnoid space, although subsequent histological examination confirmed the presence of diffuse meningeal carcinomatosis. Unfortunately the spinal cord was not always available but in 2 of the 5 spinal cords available from these 15 cases there was macroscopic evidence of meningeal tumour. Indeed, when there was only minimal macroscopic evidence of tumour in the meninges, the commonest sites in which it was seen were on 
the cauda equina and on the superior surface of the cerebellum.

Of the 23 cases in Groups $1 b$ and $2 b$ in which the tumour was restricted to the subarachnoid space the ventricles were enlarged in 9 (5 cases of primary meningeal tumours and 4 of carcinomatosis of the meninges).

The histological appearances varied widely, depending to a considerable extent on the thickness of the sheet of tumour, but the general pattern was the same throughout. There tended to be particularly severe involvement of the cerebellum (Fig. 4), the base of the brain, the brain stem and the cauda equina. Tumour cells also showed a distinct tendency to spread along perivascular spaces into the underlying parenchyma, particularly in the cerebellum (Fig. 4). In every type of tumour there was frequently direct infiltration of cranial and spinal nerve roots by tumour cells (Figs 5 and 6) and occasionally into posterior root ganglia (Fig. 7). In cases in which the meningeal tumour appeared to be present on gross inspection as multifocal deposits, microscopy revealed the additional presence of a diffuse thin sheet of tumour. Indeed the meningeal tumour often existed as a monolayer, particularly in

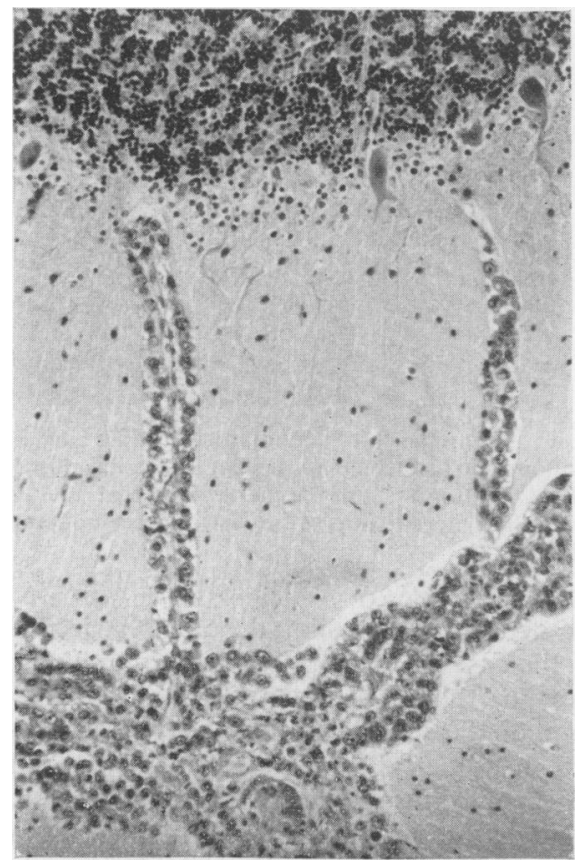

FIG. 4. Meningeal carcinomatosis. Male aged 52: carcinoma of stomach: group $2 \mathrm{~b}$. The subarachnoid space is filled with tumour cells which are also extending along perivascular spaces in the molecular layer of the cerebellum $(\mathrm{HE}, \times 100)$.

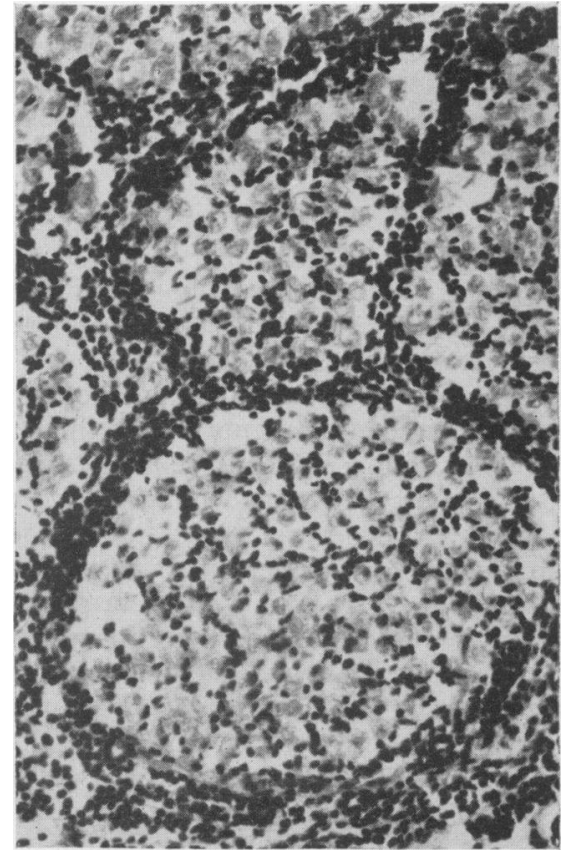

FIG. 5. Diffuse infiltration of the meninges by lymphoma. Female aged 39: systemic non-Hodgkin lymphoma:응 group $2 \mathrm{~b}$. There are tumour cells around and within nerve bundles in the spinal nerve root $(\mathrm{HE}, \times 200)$.

cases of adenocarcinoma (Fig. 8). In some cases there was also tumour on the ependyma of the ventricular system.

\section{Discussion}

The data show that a wide range of primary $\frac{3}{5}$ tumours of the CNS, in addition to medulloblastoma, may spread diffusely throughout the subarachnoid space. In the present series there were 3 . 10 examples of diffuse astrocytomatous infiltration of the meninges (including one gliosarcoma), 7 of 3 . these being anaplastic. In 2 cases of astrocytomatous $\delta$ infiltration no mass lesion in the brain or cord was $₹$ discovered post mortem. Kernohan, Woltman and o Adson (1931) were the first to describe similar cases. It is thought that such tumours arise in heterotopic o nests of glial tissue within the subarachnoid space. Polmeteer and Kernohan (1947) reported 6 cases of $\sigma$ glioblastoma and 3 of astrocytoma in their series of $N$ 22 patients with meningeal gliomatosis not due to $\mathrm{N}$ medulloblastoma. Assuming that their glioblastoma 0 corresponds to the anaplastic astrocytoma of the present series, it will be seen that the distribution of these tumour types is similar in the 2 series.

The ability of even relatively well differentiated 0 tumours occasionally to spread diffusely through the $\overline{0}$ meninges is apparent from the present series. Three 


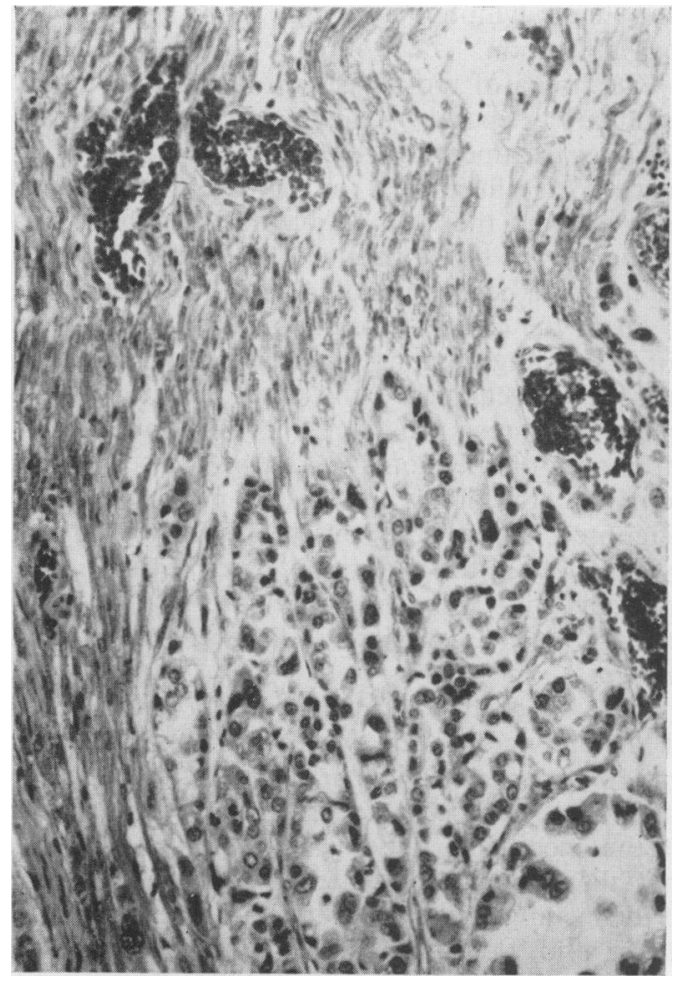

Fig. 6. Meningeal carcinomatosis. Male aged 58: carcinoma of bronchus: group $2 \mathrm{~b}$. There is a group of tumour cells within this nerve from the cauda equina $(\mathrm{HE}, \times 150)$.

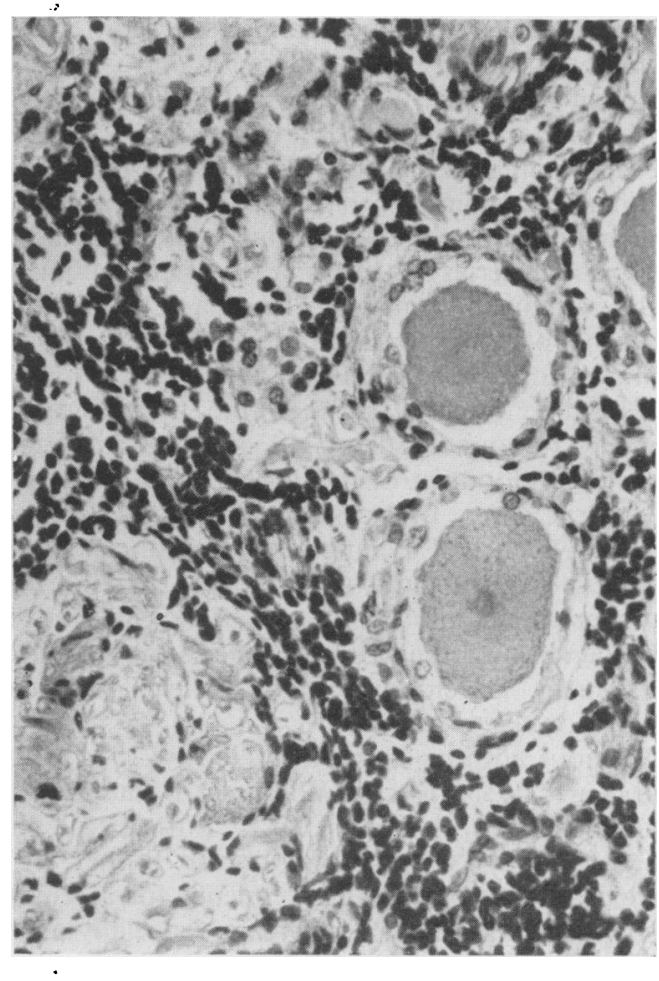

FIG. 7. Same case as Fig. 5. This posterior root ganglion is infiltrated by tumour cells $(\mathrm{HE}, \times 250)$.

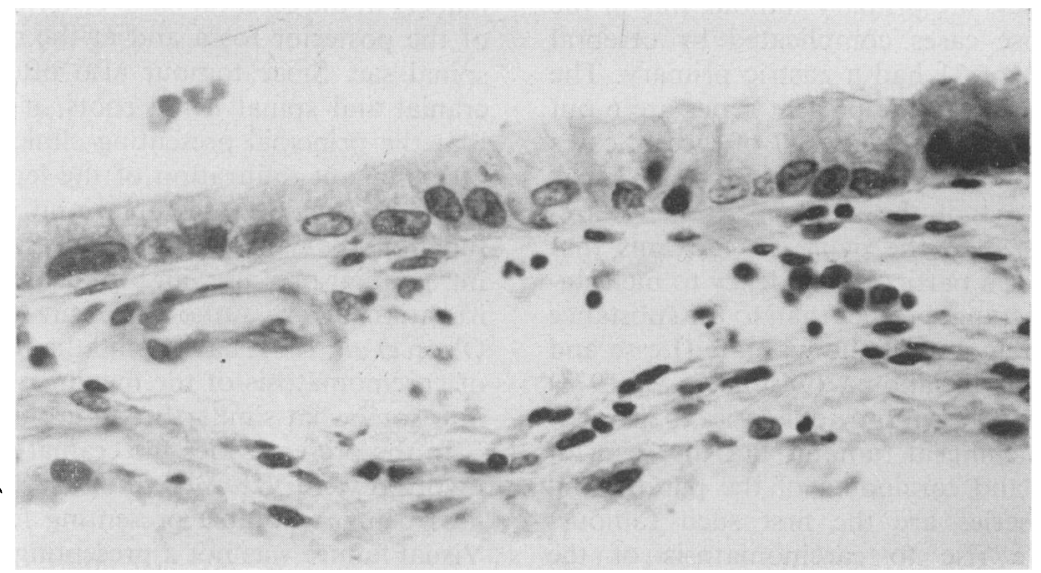

FIG. 8. Meningeal carcinomatosis. Male aged 47: carcinoma of stomach: group 2a. There is a monolayer of tumour cells on the pia covering the pons (HE, $\times 400)$. 
of the examples of astrocytomatous infiltration were at least moderately well differentiated, as was the oligodendroglioma.

Melanin-containing cells are normally found in the leptomeninges, particularly in the posterior fossa and may occasionally behave in a malignant fashion. This malignant behaviour is illustrated in 4 cases of the present series -2 examples of neurocutaneous melanosis, one of primary melanoma of the meninges and one of the rare melanotic spinal nerve root tumour (Graham et al., 1976).

The time interval between the diagnosis of a primary tumour of the CNS and the subsequent clinical development of meningeal involvement was, in general, short. In 4 cases in Group 1a there was clinical evidence of meningeal infiltration at the time of initial neurological presentation. In one case, however, there was an interval of 8 years between the diagnosis of the primary CNS tumour and the subsequent development of clinical meningeal infiltration.

Previous authors (Lesse and Netsky, 1954; Grain and Karr, 1955; Fischer-Williams, Bosanquet and Daniell, 1955; Parsons, 1972; Little, Dale and Okazaki, 1974; Olson, Chernik and Posner, 1974) have drawn attention to the tendency for carcinoma of the bronchus and of the breast, especially adenocarcinoma, to give rise to carcinomatosis of the meninges. These primary tumour types are well represented in the present series. There has been some disparity regarding the incidence of carcinoma of the stomach in past series of carcinomatosis of the meninges. Lesse and Netsky (1954) reported only 4 cases of carcinoma of the stomach in their review of 207 cases of metastatic CNS or meningeal tumour. Grain and Karr (1955) in their review of the literature found that in 33 out of 85 cases of 'pure' carcinomatosis of the meninges, without spinal or cerebral metastases, the primary tumour was in the stomach; of those cases complicated by cerebral metastases, 4 out of 31 had a gastric primary. The corresponding figures in the present series are 6 out of 18 cases and 2 out of 22 cases, 7 of these 8 cases of gastric carcinoma being adenocarcinoma. Thus, although carcinoma of the stomach does not frequently metastasize to the neuraxis, it seems that when it does it has a particular tendency to metastasize to the leptomeninges rather than to the substance of the brain. Carcinoma of the prostate (Lesse and Netsky, 1954) and melanoma (Olson et al., 1974) have also been reported in previous series to give rise to diffuse leptomeningeal tumour, but the cases of nephroblastoma and carcinoma of the parathyroid in the present series are the first such tumours reported to give rise to carcinomatosis of the meninges. Previous authors (Lesse and Netsky, 1954; Olson et al., 1974) have reported a higher incidence of systemic lymphoma giving rise to a leptomenin- $\frac{2}{3}$ geal infiltration.

Carcinoma of the breast is known to give rise toc secondary deposits many years after apparent curative treatment of the primary tumour. This property is illustrated in the present series, the interval from the time of diagnosis of the primary tumour to the development of the metastasis within $\frac{}{7}$

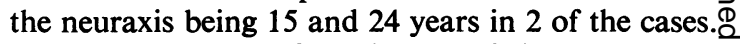
In all of the cases of carcinoma of the breast withe diffuse meningeal tumour, the primary tumour had been diagnosed before the development of neuro- logical features. However, in only one case of $\vec{\omega}$ carcinoma of the stomach and in 2 cases of carci- noma of the lungs were the primary tumourso diagnosed before the onset of the neurological 3 . dysfunction. In the majority of cases of carcinoma of the lung and stomach, particularly the latter, theos primary source was not discovered until poste mortem.

The average age of patients with primary tumours $\vec{C}$ of the nervous system with meningeal infiltration? was 36 years, 20 years younger than the average ageof patients with carcinomatosis or lymphomatosis? of the meninges. Previous authors have reported that the majority of patients with carcinomatosis of the meninges are within the $40-60$ years age ran $\vec{g} e 0$ (Lesse and Netsky, 1954; Grain and Karr, 19; Little et al., 1974; Olson et al., 1974). There was कृष marked male or female preponderance in the present series of tumours primary to the nervous system. However, there was a male preponderance (24 out of 39 cases) in the group with tumours not primary to용 the nervous system. This can be explained by the $\underset{\Rightarrow}{\Rightarrow}$ high proportion of males with carcinoma of the lung? and stomach.

As indicated above, infiltration of the meninges by tumour is most likely to be present in certain areas. namely in the cerebral basal cisterns, in the meninges 3 of the posterior fossa and at the caudal end of the spinal sac. Since tumour also often infiltrates into 3 . cranial and spinal nerve roots, it is not surprising that the principal presenting clinical manifestations $\Xi$ of malignant infiltration of the leptomeninges wereo found to include (a) cranial and spinal nerve dys- function, (b) symptoms and signs suggestive of raisedo intracranial pressure, and (c) evidence of meningeal irritation. Past authors (Grain and Karr, 1955; $\%$ Olson et al., 1974) have found that the manifestations of carcinomatosis of the meninges could be divided ज्ञ into somewhat similar broad groupings.

In the present series the cranial nerves principally involved were VII, VIII and X, with subacute deaf- $-\frac{C}{0}$ ness being a notable presenting feature in 3 cases. $\stackrel{D}{+}$ Visual failure was not a presenting feature in any of the cases, and extra-ocular palsies were not commono as presenting features. These findings are at variance $\overrightarrow{\widetilde{\Phi}}$ 
with some past reports (Fischer-Williams et al., 1955; Grain and Karr, 1955; Olson et al., 1974).

Spinal nerve dysfunction was manifested principally as areflexia, usually with some degree of weakness, in the lower extremities. The upper limbs were far less commonly affected. Sensory loss, where it was noted, was not dense and sphincter involvement was not common. Two cases in Group 1 had spastic paraplegia, found at post-mortem to be due to cord compression by leptomeningeal tumour deposits. Back pain and radicular pain were less common symptoms in patients in the present series than in some past series (Parsons, 1972; Little et al., 1974).

Symptoms and signs suggestive of raised intracranial pressure, such as headache without nuchal rigidity, vomiting, drowsiness and papilloedema were, in Group 1, associated with dense infiltration of the basal meninges and a degree of hydrocephalus. In many cases in Group 2, however, in which such clinical features were present, post-mortem examination failed to demonstrate hydrocephalus or, in fact, any evidence of raised intracranial pressure. The cause of the clinical features suggesting raised intracranial pressure in these cases is not clear, although papilloedema may have been related to local tumour infiltration around the chiasm and optic nerves.

In 9 cases in Group 2 of the present series a prominent presenting feature was the subacute development of a confusional state. This was occasionally associated with hydrocephalus but more frequently the ventricles were of normal size. The pathological basis for this confusional state was not obvious. It has been reported previously that the absence of a confusional state is supportive evidence for carcinomatosis of the meninges rather than tuberculous meningitis (Hughes, Adams and Ilbert, 1963); this is not in accordance with the present findings.

In the majority of cases where a mass lesion was present in addition to meningeal infiltration, it was the mass lesion which dominated the clinical picture. While this finding is apparently at variance with some past reports (Olson et al., 1974), it must be remembered that the present series was identified through post-mortem reports rather than from clinical diagnosis of meningeal infiltration. The majority of deaths in Groups 1a and $2 a$ was the result of the primary or secondary tumour within the brain or of the primary systemic neoplasm, the meningeal infiltrate remaining asymptomatic. In general, the density of the meningeal infiltrate found at post-mortem correlated with the degree of clinical meningeal involvement. However, it is important to note that in a number of cases, particularly where cranial nerve palsies or a confusional state were prominent, there was no macroscopic abnormality of the meninges. Microscopic invasion of the meninges and cranial nerve roots could easily be overlooked if histology is not routinely undertaken.

It was not always possible to differentiate clearly between those features related to the mass deposit and those related to the meningeal involvement; for example, a confusional state or the presence of elevated intracranial pressure could be due to either of these. In such cases, the symptoms were always attributed to the mass deposit. It was apparent, however, that cranial or spinal nerve involvement was good evidence for meningeal infiltration, and that a subacute confusional state or nuchal rigidity were also suggestive of this condition.

Neuroradiological studies were generally unhelpful in diagnosing this condition. Exclusion of a mass lesion was valuable in Groups $1 \mathrm{~b}$ and $2 \mathrm{~b}$. Demonstration of a communicating hydrocephalus in a number of cases was also helpful. Olson et al. (1974) described cases in which myelography clearly showed deposits in spinal nerves but in only one case in the present series were such deposits radiologically demonstrated. In 2 cases in Group 1a myelography revealed a spinal block, subsequently found to be due to a large leptomeningeal tumour deposit. In one case in Group 1b (astrocytomatous gliomatosis) contrast-enhanced computerized axial tomography revealed increased density in the region of the basal meninges, suggestive of a meningeal inflammatory process. In this case, however, the meningeal infiltrate was unusually dense (Fig. 1a). The main value of neuroradiology in the present series was to exclude mass lesions either within the brain, or extradurally, as the cause of symptoms and signs.

Of those cases in which the results of CSF examination were available, malignant cells were found to be present in 3 out of 14 cases in Group 1 and in 6 out of 16 cases in Group 2 . Usually the cerebrospinal fluid was examined only once. Previous surveys (Little et al., 1974; Olson et al., 1974) have shown a far higher percentage of positive results, particularly when repeated examinations of the CSF were undertaken. It is certainly the authors' recent experience that it is often necessary to examine several specimens of CSF before malignant cells are found. The findings of raised protein levels and elevated mononuclear cell counts in cases of carcinomatosis of the meninges were consistent with the established literature. Depression of the CSF glucose has been stressed in past reports (Berg, 1953; Fishman, 1963; De Vita and Canellos, 1966) to be characteristic of carcinomatosis of the meninges. However, normal glucose levels were found in 6 of the 16 cases with carcinomatosis of the meninges in the present series for which CSF examination results were available. It should be stressed that depressed CSF glucose levels were not a common finding ( 2 of 14 cases) in 
meningeal infiltration from tumours primary to the nervous system. The CSF cell count was elevated in only 6 of these 14 cases.

In those cases with a known primary tumour outside the nervous system the most common diagnostic mistake was to ascribe the neurological features entirely to the presence of either single or multiple secondary deposits within the substance of the brain. Where radiology failed to demonstrate any mass lesion either within the brain or compressing the spinal cord or cauda equina, other forms of chronic meningitis, such as tuberculous or fungal, were frequently considered in the differential diagnosis. While the finding of malignant cells within the spinal fluid is good evidence for the presence of neoplastic infiltration of the meninges, their absence does not exclude the diagnosis. In the presence of an unexplained chronic meningitic picture, the lungs and upper gastrointestinal tract should be investigated as the likeliest sites for occult neoplasm.

One case of gliomatosis of the meninges secondary to oligodendroglioma was given radiotherapy to the entire neuraxis and survived 2 months. In no other case was specific therapy directed at the meningeal involvement. Previous authors (Heathfield and Williams, 1956; Olson et al., 1974; Bramlet, Gilberti and Bender, 1976) have reported some success with radiotherapy and chemotherapy in treating carcinomatosis of the meninges. Further therapeutic trials seem justified. Ventriculoperitoneal shunting was performed in a number of cases in the present series in which hydrocephalus was demonstrated radiologically but did not substantially improve either survival time or quality of survival.

The prognosis of this condition is extremely poor. Few patients survived longer than 4 months from the onset of clinical meningeal involvement. These figures correlate well with past series (Grain and Karr, 1955; Little et al., 1974; Olson et al., 1974). There was no significant difference in survival time in Group 2 between those with and those without mass deposits in the brain.

\section{Conclusions}

1. A wide variety of malignant tumours, in addition. to medulloblastoma, have the propensity to spread diffusely through the leptomeninges; adenocarcinoma of the lung, stomach and breast is particularly likely to behave in this manner.

2. Clinical evidence of leptomeningeal involvement may be the first manifestation of systemic cancer; unexplained cranial nerve palsies, cauda equina dysfunction or a confusional state should raise the possibility of diffuse leptomeningeal tumour.

3. Clinical manifestations of diffuse leptomeningeal tumour involvement may occur with only microscopic tumour being found at post-mortem Conversely, macroscopically obvious involvement of the meninges may be present without clinical manifestations.

4. The absence of malignant cells in a singl产 specimen of CSF does not exclude the diagnosiso repeated examination of the CSF is often necessar. to demonstrate such cells.

5. Development of clinical evidence of lepto $\frac{\bar{Q}}{8}$ meningeal infiltration is of grave prognostic sige nificance, few patients surviving longer than $\mathbb{E}$ months.

\section{Acknowledgments}

We wish to thank Dr D. I. Graham, Reader in NeuraO pathology, and Dr D. Doyle, Consultant Neuropathologis for allowing us access to their neuropathological reportsig Professor J. A. Simpson for his helpful comments, and the Department of Medical Illustration, Southern Genera孞 Hospital, for providing the illustrations.

\section{References}

BERG, L. (1953) Hypoglycorrhacia of non-infective origin? diffuse meningeal neoplasm. Neurology. Minneapolis, 3 , 811.

Bramlet, D., Gilberti, J. \& Bender, J. (1976) Meningea年 carcinomatosis. Neurology. Minneapolis, 26, 287.

de Vita, V.T. \& Canellos, G.P. (1966) Hypoglycorrhacia in meningeal carcinomatosis. Cancer. New York, Philatel phia, etc., 19, 691 .

Fischer-Williams, M., Bosanquet, F.D. \& Daniell, P倠 (1955) Carcinomatosis of the meninges: a report of theos
cases. Brain, 78, 42.

FishmaN, R.A. (1963) Studies of the transport of suga between blood and cerebrospinal fluid in normal states and in meningeal carcinomatosis. Transactions of the Americas

Graham, D.I., Paterson, A., McQueen, A., Milne, J.A. \& URICH, H. (1976) Melanotic tumours (blue naevi) of spina? roots. Journal of Pathology, 118, 83.

GraIN, G.O. \& KARR, J.P. (1955) Diffuse leptomeningea carcinomatosis: clinical and pathological characteristics Neurology. Minneapolis, 5, 706.

Heathfield, K.W.G. \& Williams, J.R.B. (1956) Carcino matosis of meninges. British Medical Journal, 1, 328.

Hughes, I.E., AdAMS, J.H. \& IlberT, R.C. (1963) Invasion of the leptomeninges by tumour: the differential diagnosis from tuberculous meningitis. Journal of Neurology? Neurosurgery and Psychiatry, 26, 83.

Kernohan, J.W., Woltman, H.W. \& Adson, A.W. (1931b Intramedullary tumors of the spinal cord: a review of fifty-one cases, with an attempt at histologic classification Archives of Neurology and Psychiatry. Chicago, 25, 679. 을.

LESSE, S. \& NeTSKY, M. (1954) Metastasis of neoplasms to the central nervous system and meninges. Archives op Neurology and Psychiatry. Chicago, 72, 133.

Little, J.R., Dale, A.J.D. \& OKazaKi, H. (1974) MeningeaP carcinomatosis. Archives of Neurology. Chicago, 30, 138. $\omega$

Olson, M.E.. Chernik, N.L. \& Posner, J.B. (1974). Infiltra tion of the leptomeninges by systemic cancer. Archives of Neurology. Chicago, 30, 122.

PARSONS, M. (1972) The spinal form of carcinomatous meningitis. Quarterly Journal of Medicine, 41, 509.

Polmeteer, F.E. \& Kernohan, J.W. (1947) Meningealo gliomatosis. Archives of Neurology and Psychiatry Chicago, 57, 593. 